\title{
Ein gestörtes Zusammenspiel zwischen Europäischem IPR und dem GEK? - Probleme der Vorschaltlösung
}

\author{
Dr. Christoph Wendelstein, Konstanz
}

\section{A. Einleitung}

Wie Art. 3 GEK-VO zeigt, will der EU-Gesetzgeber das Gemeinsame Europäische Kaufrecht (GEK), welches sich in Anhang I der Verordnung findet, als fakultatives Instrument ausgestaltet wissen. Das GEK wäre aufgrund seines Verordnungscharakters zwar ein für die Mitgliedstaaten verbindlicher Rechtsakt, auf einen konkreten Kaufvertrag würde das Regelwerk gemäß Art. 3 GEK-VO jedoch nur dann zur Anwendung gelangen, wenn die Parteien des Vertrages dies vereinbart haben. Durch diese Opt in-Lösung soll ein Kaufvertragsgesetz geschaffen werden, dessen Geltung die Parteien vereinbaren müssen.

Nach dem Willen der Kommission, der in Erwägungsgrund 10 seinen Ausdruck findet, soll die Vereinbarung über die Verwendung des GEK eine Wahl sein, die innerhalb des einzelstaatlichen Rechts getroffen wird, das nach der Verordnung (EG) Nr. 593/2008 (Rom I) beziehungsweise in Bezug auf vorvertragliche Informationspflichten nach der Verordnung (EG) Nr. 864/ 2007 (Rom II) oder nach jeder anderen einschlägigen Kollisionsnorm anwendbar ist. Man mag über Sinn und Zweck, die dogmatische Umsetzbarkeit sowie die rechtspolitische Sinnhaftigkeit dieser sogenannten Vorschaltlösung streiten. Fest steht, dass sie der Wille der Kommission ist, weshalb es an dieser Stelle dabei sein Bewenden hat.

Die Folge dieser Vorschaltlösung ist es, dass man zur Anwendung des GEK nur dann gelangen kann, wenn über die Kollisionsnormen der Rom I-Verordnung, namentlich deren Art. 3, 4 und 6, das Recht eines Mitgliedstaates zur Anwendung berufen wird. Demgegenüber kann man mittels der vorgeschalteten Kollisionsnormen der Rom II-Verordnung nur in Bezug auf vorvertragliche Informationspflichten zur Anwendung des GEK gelangen, wenn diese das Sachrecht eines Mitgliedstaates zur Anwendung berufen. Nur in diesen Fällen besteht die Möglichkeit einer Einwahl in das GEK in Form einer sogenannten „Binnenwahl“, als Auswahl einer von zwei Sachrechtsordnungen, die über die Kollisionsnormen des Internationalen Privatrechts zur Anwendung berufen sind.

Funktionieren kann dieses System der Vorschaltlösung nur, wenn die durch das GEK geregelten Ansprüche in den Anwendungsbereich der Rom I-Verordnung, der Rom II-Verordnung (jedoch ausschließlich in Bezug auf vorvertragliche Informationspflichten) oder jeder anderen Kollisionsnorm fallen. Fände sich im GEK hingegen eine Anspruchsgrundlage, die in den Anwendungsbereich der Rom II-Verordnung fallen würde und keinen Bezug auf vorvertragliche Informationspflichten aufweist, könnte diese in keinem Fall zur Anwendung gelangen, da die Einwahl in das GEK - jedenfalls sofern man den Wortlaut des Erwägungsgrundes 10 zum GEK ernst nimmt - nicht möglich ist, weil diese dann nicht „innerhalb des einzelstaatlichen Rechts getroffen wird, das nach der Verordnung (EG) Nr. 593/2008“ anwendbar ist. Da kein Bezug zu vorvertraglichen Informationspflichten besteht, würde die Einwahl auch nicht innerhalb des einzelstaatlichen Rechts getroffen, das in Bezug auf vorvertragliche Informationspflichten nach der Rom II-Verordnung auf außervertragliche Schuldverhältnisse zur Anwendung berufen ist. Auch die dritte Alternative des Erwägungsgrunds 10 zum GEK („oder nach jeder anderen einschlägigen Kollisionsnorm“) würde nicht eingreifen, da der durch das GEK geregelte Anspruch ja an sich in den Anwendungsbereich der Rom II-Verordnung fallen würde und damit nicht nach einer „anderen“ Kollisionsnorm anzuknüpfen ist, sondern eben nach dem Kollisionsrechtsregime der Rom II-Verordnung.

Die Kommission scheint somit davon auszugehen, dass alle Sachverhalte, die keinen Bezug zu vorvertraglichen Informationspflichten aufweisen und im GEK geregelt sind, von dem Kollisionsrechtsregime der Rom I-Verordnung oder anderen, außerhalb der Rom II-Verordnung liegenden Kollisionsnormen, erfasst werden. Dies wäre aber nur dann der Fall, wenn es sich bei diesen Sachverhalten bzw. den aus diesen resultierenden Ansprüchen um vertragliche Ansprüche im Sinne des Art. 1 Abs. 1 Rom I handeln würde, da sie anderenfalls schon nicht in den Anwendungsbereich der Rom I-Verordnung fallen würden. Problematisch ist diese Sichtweise der Kommission insbesondere im Spannungsverhältnis zwischen vertraglicher und deliktischer Haftung, da in diesem „Graufeld“ eine eindeutige Qualifikation von Ansprüchen naturgemäß schwer fällt und daher häufig umstritten ist. Den Auswirkungen dieser Qualifikationsprobleme auf die Vorschaltlösung wird im Rahmen dieses Beitrags nachgegangen. 


\section{B. Qualifikationsfragen}

\section{Unproblematische Fälle}

Sicher ist, dass die in Art. 123 und Art. 91 GEK geregelten kaufrechtlichen Primäransprüche von Käufer und Verkäufer, also insbesondere die Abnahme der Kaufsache und Bezahlung des Kaufpreises einerseits und die Lieferung einer vertragsgemäßen Kaufsache andererseits, als vertraglich im Sinne der Rom I zu qualifizieren sind. Dies resultiert schon daraus, dass sich gemäß Art. 12 Abs. 1 lit. b) Rom I die Erfüllung der durch den Vertrag begründeten Verpflichtungen nach der auf den Vertrag anwendbaren Sachrechtsordnung beurteilt. Wie sich aus Art. 12 Abs. 1 lit. c) Rom I ergibt, sind auch die Sekundäransprüche von Käufer und Verkäufer wegen der vollständigen oder teilweisen Nichterfüllung der Primärpflichten des Kaufvertrages als vertraglich zu qualifizieren. In diesem Bereich sind daher keine Probleme bezüglich der von der Kommission gewählten Vorschaltlösung zu erwarten.

\section{Problematische Fälle}

Problematisch ist hingegen die Qualifikation von Schadensersatzansprüchen des Käufers oder des Verkäufers wegen eines Sach- oder Personenschadens. ${ }^{1}$ Gemäß Art. 2 lit. g) GEK umfasst der Begriff des Schadensersatzes einen Geldbetrag, zu dem eine Person als Entschädigung für einen erlittenen Verlust oder einen körperlichen oder sonstigen Schaden berechtigt sein kann. Nach Art. 179 Nr. 2 GEK beträgt die lange Verjährungsfrist bei Schadensersatzansprüchen wegen Personenschäden dreißig Jahre. Insbesondere in diesen beiden Regelungen kommt zum Ausdruck, dass die Kommission davon ausgeht, dass Schadensersatzansprüche wegen der Schädigung einer Person in den Anwendungsbereich des GEK fallen. Nach Erwägungsgrund 27 S. 1 zum GEK unterliegen alle vertraglichen und außervertraglichen Sachverhalte, die nicht im GEK geregelt sind, dem außerhalb des GEK bestehenden innerstaatlichen Recht, das nach Maßgabe der Rom I- und Rom II-Verordnungen oder nach sonstigen einschlägigen Kollisionsnormen anwendbar ist. Zu diesem Bereich zählt gemäß Erwägungsgrund 27 S. 2 unter anderem das Deliktsrecht. Da in Art. 2 lit. g) und Art. 179 Nr. 2 GEK aber auch die Personenschäden erwähnt sind, scheint die Kommission der Auffassung zu sein, dass Ansprüche aus Verletzung der körperlichen Integrität vertraglicher und nicht deliktischer Natur sind. Anderenfalls wären derartige Ansprüche der über die Rom IIVerordnung zur Anwendung berufenen Sachrechtsordnung unterstellt und eine sachrechtliche Einwahl in das GEK würde gemäß Erwägungsgrund 10 zum GEK ausscheiden. Ob diese Sichtweise überzeugt, wird im Folgenden zu klären sein.

Probleme bereitet die Qualifikation von Ansprüchen aus Schädigungen der Person insbesondere deshalb, weil der Schutz der persönlichen Integrität in den modernen europäischen Sachrechtsordnungen (zumindest auch) eine Domäne des Deliktsrechts ist. Bevor der Frage nach der Qualifikation derartiger Ansprüche näher nachgegangen werden kann, ist daher zunächst zu klären, wo die Grenze zwischen Vertrag und Delikt auf der Ebene des europäischen Kollisionsrechts überhaupt verläuft. Erst im
Anschluss an diese Feststellung ist eine Einordnung derartiger Ansprüche in das Kollisionsrechtsregime der Rom I- und/oder Rom II-Verordnung möglich.

\section{Abgrenzung von Vertrag und Delikt im Europäischen Kollisionsrecht}

Bereits an anderer Stelle wurde gezeigt, dass die Grenze zwischen Vertrag und unerlaubter Handlung auf der Ebene des Europäischen Kollisionsrechts keinesfalls identisch ist mit derjenigen im deutschen Sachrecht. ${ }^{2}$ Dies ist nicht verwunderlich, da die Qualifikation von Rechtsfragen im Anwendungsbereich von Rom I und Rom II nicht davon abhängt, an welchen Stellen - die durch historische Zufälligkeiten bedingt sein können ${ }^{3}$ - die beteiligten Sachrechte, sei es die lex causae oder die lex fori, den betroffenen Fragenkreis regeln. Vielmehr hat im Anwendungsbereich von Rom I und Rom II die Qualifikation autonom von der jeweiligen Sachrechtsordnung zu erfolgen, ${ }^{4}$ da beispielsweise die Systembegriffe „Vertrag“ und „unerlaubte Handlung“ im europäischen Kollisionsrecht und die Systembegriffe „Vertrag“ und „unerlaubte Handlung“, welche die Sachrechtsordnungen aufgliedern, nicht zwangsläufig identisch sind, stammen sie doch bereits von unterschiedlichen Gesetzgebern. ${ }^{5}$ Unabhängig von dieser Feststellung sind für die Beantwortung der Qualifikationsfrage nicht die Systembegriffe der jeweiligen lex fori oder lex causae, sondern vielmehr der mit einer Rechtsnorm verfolgte Zweck mit den daraus implizierten kollisionsrechtlichen Interessen entscheidend. ${ }^{6}$ Die Ziehung einer Grenze zwischen Vertrag und Delikt im Europäischen Kollisionsrecht hat somit autonom von vergleichbaren Grenzziehungen in den Sachrechtsordnungen der Mitgliedstaaten zu erfolgen. Auf der Ebene des Kollisionsrechts muss sie anhand der unterschiedlichen Funktionen dieser beiden Rechtsinstitute autonom vom Sachrecht bestimmt werden. Denn aus der unterschiedlichen Funktion von Vertrag

1 Gemäß Art. 5 CISG fällt die Haftung für derartige Schäden nicht in den Anwendungsbereich des UN-Kaufrechts. In der Folge beurteilt sich die Haftung für Tod oder Körperverletzung nicht nach dem CISG, sondern nach der über das Internationale Privatrecht zur Anwendung berufenen Sachrechtsordnung. Vgl. Kieninger, in: Schulte-Nölke/Zoll/Jansen/ Schulze, Der Entwurf für ein optionales europäisches Kaufrecht, 2012, 205, 211; Köhler, Die Haftung nach UN-Kaufrecht im Spannungsverhältnis zwischen Vertrag und Delikt, 2003, S. 134.

2 Wendelstein, Kollisionsrechtliche Probleme der Telemedizin, 2012, S. 135-166.

3 Vgl. Mankowski, IPrax 2003, 127, 133; siehe auch Generalanwalt Francis G. Jacobs, Schlussanträge in Rs. C-26/91 v. 8.4.1992, Slg. 1992, I-3977, I-3985 Nr. 26.

4 Erwägungsgrund Nr. 11 zu Rom II; Dutta, IPrax 2009, 293, 293; Thorn, in: Palandt, (Fn. 4), Vorbemerkung zu Rom I Rn. 3 i.V.m. Art. 3 EGBGB Rn. 9a; Dickinson, The Rome II Regulation: The Law Applicable to Non-Contractual Obligations, 2008, Rn. 3.86, 3.243, 4.06.; Martiny, in: MüKo, 5. Auflage 2010, Vor Art. 1 Rom I Rn. 15; McLean/Beevers, Conflict of Laws, 7. Auflage 2009, Rn. 14-012; Von Hein, in: Rauscher (Hrsg.), EuzPR/EuIPR, Bearbeitung 2011, Art. 1 Rom I Rn. 5.

5 Vgl. Schurig, Kollisionsnorm und Sachrecht, 1981, S. 215, 219 f.

6 Hierdurch wird die Interessenjurisprudenz in das IPR übernommen. Wie die materiell-privatrechtliche Gerechtigkeit (nach der Lehre der Interessenjurisprudenz) auf Feststellung, Bewertung und Abwägung von Interessen beruht, beruht auch die internationalprivatrechtliche Gerechtigkeit darauf, wenngleich es dabei um die Feststellung, Bewertung und Abwägung kollisionsrechtlicher Interessen geht. Vgl. dazu Kegel, in: FS Lewald, 1953, S. 259 ff., insb. S. $261 \mathrm{ff}$. 
und Delikt resultieren unterschiedliche kollisionsrechtliche Interessenlagen, die dazu geführt haben, dass für vertragliche und deliktische Ansprüche überhaupt unterschiedliche Statute ausgebildet wurden.

Im Folgenden wird daher zunächst herausgearbeitet, welche Funktionen die Rechtsinstitute Vertrag und Delikt bei EU-autonomem Verständnis besitzen. Problematisch ist dabei, dass weder die Rom I- noch die Rom II-Verordnung eine Legaldefinition des Vertrages beziehungsweise der unerlaubten Handlung enthalten. Auch das GEK hilft insoweit nicht weiter: Zwar definiert die Kommission in Art. 2 lit. a) GEK den Vertrag als „eine Vereinbarung, die darauf abzielt, Verpflichtungen oder andere rechtliche Wirkungen herbeizuführen“. Diese Definition soll jedoch gemäß Art. 2 GEK nur für die Zwecke des GEK gelten, weshalb sie zur Abgrenzung von Vertrag und Delikt auf der Ebene des Europäischen Kollisionsrechts nicht hilfreich ist.

\section{a) Unterscheidung zwischen Vertrag und Delikt durch den EuGH}

Der EuGH hat im Rahmen der Auslegung von Art. 5 Nr. 1 und 3 EuGVO einen Vertragsbegriff entwickelt. Dieser lässt sich grundsätzlich auch zur Abgrenzung von Vertrag und Delikt auf der Ebene des europäischen Kollisionsrechts fruchtbar machen. ${ }^{7}$ Die Übertragung der Rechtsprechung des EuGH zur Auslegung der Begriffe „Vertrag“ und „unerlaubte Handlung“ in Art. 5 Nr. 1 und Nr. 3 EuGVO auf die Rom I- und II-Verordnung ist jedoch nur in beschränktem Maße hilfreich, da der autonome Vertragsbegriff des EuGH bislang stark unterentwickelt ist. So verlangt der EuGH als Voraussetzung für einen Vertrag eine „freiwillig eingegangene Verpflichtung“ der einen Partei gegenüber der anderen. ${ }^{8}$ An einem Vertrag fehlt es daher in Situationen „in der es an einer von einer Partei gegenüber einer anderen freiwillig eingegangenen Verpflichtung fehlt“.${ }^{9}$ Demgegenüber definiert der EuGH den autonomen Begriff der „unerlaubte Handlung“ im Sinne des Art. 5 Nr. 3 EuGVO schlicht negativ in dem Sinn, dass er sich „auf alle Klagen bezieht, mit denen eine Schadenshaftung geltend gemacht wird und die nicht an einen Vertrag im Sinne von Art. 5 Nr. 1 EuGVO anknüpfen “. ${ }^{10}$

Die Aussage des EuGH, dass es an einem Vertrag in einer Situation fehlt, „,in der es an einer von einer Partei gegenüber einer anderen freiwillig eingegangenen Verpflichtung fehlt", steht zwar nicht im Widerspruch zum allgemeinen Vertragsbegriff, ist aus der Sicht der materiellen Rechtsordnungen und des Kollisionsrechts aber noch zurückgeblieben. ${ }^{11}$ Er legt nicht die hinreichenden Bedingungen zur Qualifikation eines Anspruchs als vertraglich fest. Die Interpretationen des Art. 5 Nr. 1 und Nr. 3 EuGVO durch den EuGH geben folglich lediglich die Mindestanforderungen an einen Vertrag vor, sind darüber hinaus aber für die Abgrenzung von Rom I und II wenig hilfreich. ${ }^{12}$ Sie können daher allenfalls als eine Art Leitlinie fungieren. ${ }^{13}$

Dennoch steht man bei der Bestimmung des autonomen kollisionsrechtlichen Vertragsbegriffs nicht mit leeren Händen da: Erstens kennt man die Minimalvoraussetzungen, nämlich das Erfordernis einer „freiwillig eingegangenen Verpflichtung“. Zweitens lassen sich aus der Funktion von Vertrag und Delikt auf der Ebene der Sachrechte und der daraus implizierten kollisionsrechtlichen Interessenlage gewisse Rückschlüsse ziehen.
Drittens lassen sich aus dem Zweck, mit dem der Begriff des Vertrags in der Rom I-Verordnung verwendet wird, gewisse Anhaltspunkte für das ihr zugrunde liegende Vertragsverständnis ableiten. ${ }^{14}$

\section{b) Rückschlüsse aus der Funktion von Vertrag und Delikt auf der Ebene des Sachrechts}

Sinn und Zweck eines Vertrages ist ein irgendwie gearteter Rechts- oder Gütertransfer von einer Vertragspartei auf die jeweils andere. ${ }^{15}$ Das konkrete Vertragsversprechen ist zukunftsorientiert: Jede Vertragspartei verspricht für die Zeit nach dem Vertragsschluss etwas zu tun oder zu unterlassen. Die Befugnis der Vertragsparteien, sich für ihr versprochenes Tun oder Unterlassen ein eigenes Regelwerk zu schaffen, ist Ausfluss der Privatautonomie. Durch die grundsätzliche Anerkennung und Achtung des durch die Parteien geschaffenen Regelwerks wird für den Zeitraum der Vertragsdurchführung ein vom Gesetz grundsätzlich unabhängiger und zwischen den Vertragsparteien verbindlicher Verhaltenskodex geschaffen, welcher an der Verwirklichung des Äquivalenzinteresses ausgerichtet ist. ${ }^{16}$

Im Gegensatz hierzu dient das Deliktsrecht der Absicherung des status quo an Rechtsgütern, die einer Person durch die

7 Wie hier von Hein, (Fn. 4), Art. 1 Rom I Rn. 5; Bitter, IPRax 2008, 96 ff.; Dutta, IPRax 2009, $295 \mathrm{f}$.

8 EuGH, Rs. C-26/91, Urt. v. 17.6.1992, Slg. 1992, I-3990, I-3994 Rn. 15; Rs. C-51/97, Urt. v. 27.10.1998, Slg. 1998, I-6534, I-6542 Rn. 17; Rs. C-334/00, Urt. v. 17.9.2002, Slg. 2002, I-7383, I-7393 Rn. 23; Rs. C-265/02, Urt. v. 5.2.2004, Slg. 2004, I-1546, I-1555 Rn. 24; Rs. C-27/02, Urt. v. 20.1.2005, Slg. 2005, I-499, I-512 Rn. 29; Schack, Internationales Zivilverfahrensrecht, 5. Auflage 2010, Rn. 292; Kropholler/ Von Hein, Europäisches Zivilprozessrecht, 9. Auflage 2011, Art. 5 Rn. 9.

9 EuGH, Rs. C-51/97, Urt. v. 27.10.1998, Slg. 1998, I-6534, I-6542 Rn. 17.

10 EuGH, NJW 1988, 3088, 3089; Rs. C-51/97, Urt. v. 27.10.1998, Slg. 1998, I-6534, I-6542 Rn. 22; Rs. C-96/00, Urt. v. 11.7.2002, Slg. 2002, I-6384, I-6398 Rn. 33; Rs. C-167/00, Urt. v. 1.10.2002, Slg. 2002, I-8126, 8139 Rn. 36; Rs. C-27/02, Urt. v. 10.1.2005, Slg. 2005, I-499, I-512 Rn. 29.

$11 \mathrm{Zu}$ den Gründen hierfür vgl. Wendelstein, (Fn. 2), S. 147 f.

12 Lehmann, Der Anwendungsbereich der Rom I-Verordnung - Vertragsbegriff und vorvertragliche Rechtsverhältnisse, in: Ferrari/Leible (Hrsg.), Ein neues Internationales Vertragsrecht für Europa - Der Vorschlag für eine Rom-I Verordnung, 2007, 17, 27; Wendelstein, (Fn. 2), S. 148.

13 Von Hein, in: Rauscher, (Fn. 4), Art. 1 Rom I Rn. 5.

14 Vgl. Lehmann, (Fn. 12), 17, 28; Wendelstein, (Fn. 2), S. 149.

15 Zweigert/Kötz, Einführung in die Rechtsvergleichung, 3. Auflage 1996, $\$ 40$ I, S. 598; Medicus/Lorenz, Schuldrecht I - Allgemeiner Teil, 20. Auflage 2012, Rn. 117; von Bar/Drobnig, Study on Property Law and Noncontractual Liability Law as they relate to Contract Law, 2004, http://ec.eu ropa.eu/consumers/cons_int/safe_shop/fair_bus_pract/cont_law/study. pdf; zuletzt abgerufen am 12.2.2013, Rn. 38; vgl. insoweit auch Picker, JZ 1987, 1041, 1044; Leicht, Die Qualifikation der Haftung von Angehörigen rechts- und wirtschaftsberatender Berufe im grenzüberschreitenden Dienstleistungsverkehr, 2001, S. 153; Unberath, Die Vertragsverletzung, 2007, S. 184; Wendelstein, (Fn. 2), S. 149.

16 Vgl. dazu Schlechtriem, in: BMJ (Hrsg.), Gutachten und Vorschläge zur Überarbeitung des Schuldrechts, Band II, 1981, S. 1591, S. 1608; Wendelstein, (Fn. 2), S. 149; Aus europäischer Perspektive Schwartze, Europäische Sachmängelgewährleistung beim Warenkauf, 2003, S. 63; Hoffmann, Koordination des Vertrags- und Deliktsrecht in Europa, 2006, S. 26 (nach rechtsvergleichender Betrachtung der Sachrechtsordnungen von Frankreich, Italien, England, den Niederlanden und Deutschland); Decku, Zwischen Vertrag und Delikt, 1995, S. 150 (nach rechtsvergleichender Betrachtung der Sachrechtsordnungen von England und Deutschland); Weir, in: Trunc (Hrsg.), International Encyclopedia of Comparative Law XI/2, 1986, Chapter 12, S. 5. 
Rechtsordnung bereits zugewiesen sind, gegenüber Jedermann. Das Deliktsrecht besteht damit unabhängig von einer privatautonomen Vereinbarung. Zur Erreichung dieses Schutzes enthalten die Sachrechte Regeln, die auf den Integritätsschutz abzielen. Sinn und Zweck des Deliktsrechts ist somit nicht die Absicherung eines irgendwie gearteten Gütertransfers sondern einzig der Schutz des Integritätsinteresses. ${ }^{17}$ Im Mittelpunkt des Deliktsrechts stehen demgemäß nicht die Anerkennung und der Schutz eines parteiautonomen Verhaltenskodex, sondern der Schutz der Rechtsgüter einer Person. Bei der Haftung aus unerlaubter Handlung handelt es sich um eine staatlich aufgezwungene Haftung, ${ }^{18}$ die - unter gewissen Voraussetzungen - unabhängig von einem irgendwie gearteten Parteiwillen kraft autoritärer, staatlicher Rechtssetzung mit Eintritt eines Schadensereignisses entsteht. ${ }^{19}$ Sinn und Zweck dieser staatlich aufgezwungenen Haftung ist die „Sicherung jener natürlichen Macht des Menschen über sich selbst gegen fremde Einmischung ". ${ }^{20}$ Dem Deliktsrecht obliegt folglich der Schutz der einer Person durch die Rechtsordnung zugewiesenen Rechtsgüter als Grundlage ihrer freien, parteiautonomen Willensbildung. ${ }^{21}$ Es dient der Schaffung „der Bedingungen, unter denen die Willkür des einen mit der Willkür des anderen nach einem allgemeinen Gesetze der Freiheit zusammen vereinigt werden kann".22

Spiegelt man die aus dieser Funktionsverteilung zwischen Vertrag und Delikt resultierende sachrechtliche Interessenlage auf die Ebene des Kollisionsrechts, erhält man die dem Vertragsstatut einerseits und dem Deliktsstatut andererseits zugrundeliegende kollisionsrechtliche Interessenlage. Nach dieser Spiegelung obliegt dem Vertragsstatut die Aufgabe, das Äquivalenzinteresse, also das Interesse eines Vertragspartners am Erhalt der vertraglich vereinbarten Primärleistung in der im Vertrag vereinbarten Form, kollisionsrechtlich zu schützen. ${ }^{23}$ Demgegenüber besitzt das Deliktsstatut die Aufgabe, die Integritätsinteressen, also das Interesse einer Person an der Unversehrtheit ihres status quo, zu schützen. ${ }^{24}$

\section{c) Funktionaler kollisionsrechtlicher Vertragsbegriff}

Diese Sichtweise steht auch im Einklang mit der Funktion des kollisionsrechtlichen Vertragsbegriffs. Funktional betrachtet ist der Vertragsbegriff der Schlüssel zur Abgrenzung der Anwendungsbereiche von Rom I und II, da alles was nicht vertraglich ist, als außervertragliches Recht grundsätzlich in den Anwendungsbereich der Rom II-Verordnung fällt. ${ }^{25}$ Somit lässt sich vermuten, dass sich aus einem funktionalen Vergleich der Kollisionsregeln der Rom-I und II-Verordnungen Rückschlüsse auf den Vertragsbegriff ziehen lassen.

Den Kristallisationspunkt der Rom I-Verordnung bildet die Parteiautonomie. ${ }^{26}$ Selbst im Verbraucherkollisionsrecht ist eine Rechtswahl nicht ausgeschlossen, wie Art. 6 Abs. 2 Rom I zeigt. Parteiautonomie bedeutet - wie die Privatautonomie als ihr sachrechtliches Gegenstück - die Möglichkeit zur privaten Selbstgestaltung. Sie ist nur dort sinnvoll und angebracht, wo die Parteien ihre Verhältnisse selber regeln (können). ${ }^{27}$

Anders verhält es sich im Anwendungsbereich der Rom IIVerordnung: Zwar ist auch dort eine Rechtswahl für außervertragliche Schuldverhältnisse unter Umständen zulässig. Gemäß Art. 14 Abs. 1 Rom II ist diese jedoch grundsätzlich auf den Zeit- raum nach Eintritt des Ereignisses, durch welches das außervertragliche Schuldverhältnis entstanden ist, beschränkt. ${ }^{28}$ Die Rechtswahl stellt somit kein zentrales Element der Rom II-Verordnung dar. ${ }^{29}$

Haben die Parteien keine Rechtswahl getroffen, stellt Art. 4 Rom I auf das Recht des Staates ab, in dem die Partei, welche die charakteristische Leistung zu erbringen hat, ihren gewöhnlichen Aufenthalt oder ihre Hauptverwaltung hat. Ganz anders

17 Vgl. dazu aus europäischer Perspektive Schwartze, (Fn. 16), S. 63 f.; auch Hoffmann, (Fn. 16), S. 26 f. (nach rechtsvergleichender Betrachtung der Sachrechtsordnungen von Frankreich, Italien, England, den Niederlanden und Deutschland); ferner Von Bar/Drobnig, (Fn. 15), Rn. 38; Decku, (Fn. 16), S. 150 (nach rechtsvergleichender Betrachtung der Sachrechtsordnungen von England und Deutschland); Weir, (Fn. 16), Chapter 12, S. 5; sowie Zweigert/Kötz, (Fn. 15), \$ 40 I, S. 598; Giesen, International Medical Malpractice Law, 1988, \$2 Rn. 8; Leicht, (Fn. 15), S. 153; Schlechtriem, in: BMJ, (Fn. 16), S. 1591, S. 1608; Stauch, The Law of Medical Negligence in England and Germany, 2008, S. 6; Medicus, in: FS Kern, 313, 327; Wendelstein, (Fn. 2), S. 150.

18 Kerameus, Vertrag und Delikt in der Rechtsprechung des Gerichtshofs der Euopäischen Gemeinschaften, 2002, S. 1.

19 Dobbs, Law of Torts, 2000, \$3 S. 5; Prosser/Keeton, Prosser and Keeton on the Law of Torts, 5. Auflage 1984, $\$ 92$ S. 655; vgl. auch Stein, Gruchot 28 (1884), 406, 415

20 Von Savigny, System des heutigen Römischen Rechts, Band I, 1840, S. 336.

21 Von Savigny, (Fn. 20), S. 336 f.; Peukert, Güterzuordnung als Rechtsprinzip, 2008, S. 248; Larenz/Canaris, Lehrbuch des Schuldrechts II/2, 13. Auflage 1994, S. 350; Brüggemeier, Deliktsrecht, 1986, S. 70 f.; Von Bar/Drobnig, (Fn. 15), Rn. 38; Auch die Verfasser des BGB sahen die Aufgabe des Deliktsrechts darin, „die Rechtskreise der Einzelnen, innerhalb deren sie ihre individuelle Freiheit entfalten und ihre Interessen verfolgen dürfen, von einander abzugrenzen“, Mugdan, Die gesamten Materialien zum Bürgerlichen Gesetzbuch für das Deutsche Reich, Band II, 1979, S. 1073 Rn. 2712; vgl. auch Tunc, in: Trunc (Hrsg.), International Encyclopedia of Comparative Law XI/1, 1983, Introduction, S. 14 f. Rn. 22; Spickhoff, in: Soergel (Hrsg.), Band 12, 13. Auflage 2005, Vor $\$ 823$ BGB Rn. 29.

22 Kant, Die Metaphysik der Sitten, 2. Auflage 1798, S. 336, Orginalpaginierung A 33 B 33, 34.

23 Von Bar, Internationales Privatrecht, Zweiter Band, 1991, Rn. 556; Canaris, in: FS Larenz, 27, 109; bei autonomer funktionaler Qualifikation auch Hoffmann, (Fn. 16), S. 172 und Bröcker, Möglichkeiten der Differenzierten Regelbildung im internationalen Deliktsrecht, 1967, S. 210; Brödeman/ Wegen, in: Prütting/Wegen/Weinreich (Hrsg.), BGB, 5. Auflage 2010, Art. 12 Rom I Rn. 34; Spellenberg, in: MüKo, (Fn. 4), Art. 12 Rom I Rn. 79; Stoll, in: FS Georgiades, 941, 958; Wendelstein, (Fn. 2), S. 150 f.

24 Vgl. Schurig, in: FS Heldrich, 1021, 1027; Brödeman/Wegen, in: Prütting/ Wegen/Weinreich, (Fn. 23), Art. 12 Rom I Rn. 34; Von Bar, (Fn. 23), Rn. 556; Canaris, in: FS Larenz, 27, 109; Spellenberg, in: MüKo, (Fn. 4), Art. 12 Rom I Rn. 79; Wendelstein, (Fn. 2), S. 151; bei autonomer funktionaler Qualifikation auch Hoffmann, (Fn. 16), S. 172 und Bröcker, (Fn. 23), S. 210.

25 Besonders deutlich wird dies bei den Ausführungen von Plender/Wilderspin, The European Private International Law of Obligations, 3. Auflage 2009, Rn. 2-001-2-077 die unter der Überschrift „The Dividing Line Between Rome I and Rome II" stehen.

26 Siehe dazu Art. 3 R I; Vgl. auch Erwägungsgrund Nr. 11 zu Rom I, der die Rechtswahl als Eckstein des internationalen Vertragsrechts bezeichnet.

27 Lehmann, (Fn. 12), 17, 29; ähnlich Mankowski, IPRax 2003, 127, 131.

28 Eine Ausnahme von diesem Grundsatz stellt Art. 14 Abs. 1 1. b) Rom II dar, der es kommerziell tätigen Parteien unter gewissen, engen Voraussetzungen gestattet eine Rechtswahl für außervertragliche Ansprüche auch vor Eintritt des schadensbegründenden Ereignisses zu vereinbaren. Dass es sich bei dieser Regelung um eine Ausnahme handelt, ergibt sich zum einen aus ihrem engen Anwendungsbereich und der Struktur der Rom II-Verordnung. Eine planende, vorausschauende Rechtswahl durch die Parteien ist bei außervertraglichen Schuldverhältnissen praktisch nur selten möglich und rechtlich weitgehend auch gar nicht gestattet. Vgl. dazu Lehmann, (Fn. 12), 17, 29.

29 Rusworth/Scott, LMCLQ 2008, 274, 300; Wendelstein, (Fn. 2), S. 156. 
entscheidet hingegen die Rom II-Verordnung: Gemäß ihres Art. 4 Abs. 1 wird im Grundsatz das Recht des Staates, in dem der Schaden eintritt oder einzutreten droht, zur Anwendung berufen. Hierdurch soll ein gerechter Ausgleich zwischen den kollisionsrechtlichen Interessen des Schädigers und des Geschädigten erreicht werden. ${ }^{30}$ Als Anknüpfungsalternativen wurden das Recht am Handlungsort und ein einseitiges Wahlrecht des Geschädigten vorgeschlagen. Die jetzige Anknüpfung an das Recht am Erfolgsort ist nach Auffassung der Kommission ein interessengerechter Kompromiss zwischen diesen beiden extremen Anknüpfungsalternativen. ${ }^{31}$ Mit der Regelung des Art. 4 Abs. 1 Rom II ist daher auch ein gewisser Grad an Opferschutz beabsichtigt. Besonders deutlich kommt dieser Gesichtspunkt in der in Art. 7 und Art. 6 Abs. 3 lit. b Rom II vorgesehenen Möglichkeit einer einseitigen Rechtsbestimmung durch den Geschädigten zum Ausdruck. Bezüglich Sicherheits- und Verhaltensregeln ist hingegen gemäß Art. 17 Rom II stets das Recht am Ort des schädigenden Ereignisses faktisch zu berücksichtigen, damit der potentielle Deliktstäter die einschlägigen Sicherheits- und Verhaltensregeln voraussehen und sein Handeln an diesen ausrichten kann.

Zusammenfassend bedeutet dies: Zentrales Element der Rom I-Verordnung ist die Anerkennung der Privatautonomie auf der Ebene des Kollisionsrechts. Demgegenüber besteht der Sinn und Zweck der Kollisionsregeln der Rom II-Verordnung darin, einen angemessenen Ausgleich der kollisionsrechtlichen Interessen von Täter und Opfer sowie die Voraussehbarkeit der anwendbaren Sicherheits- und Verhaltensregeln zu erreichen. Für die Qualifikation einer Sachnorm als vertraglich oder deliktisch ergibt sich daraus Folgendes: Geht es schwerpunktmäßig um die Anerkennung und Achtung der Privatautonomie auf der Ebene des Kollisionsrechts, ist vom Vorliegen eines vertraglichen Anspruchs auszugehen, so dass der Anwendungsbereich von Rom I eröffnet ist. ${ }^{32}$ Dahingegen hat eine vertragliche Qualifikation in Fällen, in denen es nicht um Ansprüche aus der Verletzung einer parteiautonom vereinbarten Verpflichtung geht, zu unterbleiben.

Zur Überprüfung, ob Ansprüche aus der Verletzung einer parteiautonomen Vereinbarung resultieren oder unabhängig von einer solchen kraft Gesetzes bestehen, ist festzustellen, ob bei gleicher Faktizität der Ereignisse ohne freiwillig eingegangene Verpflichtung keine Haftung bestehen würde. ${ }^{33}$ Nur dann ist die privatautonome Abrede conditio sine qua non für die Haftung, da sich nur dann die kollisionsrechtliche Interessenlage der Beteiligten durch den Vertragsschluss derart verändert hat, dass eine Zuordnung zum Kollisionsrechtssystem der Rom I-Verordnung sachgerecht erscheint. Gelangt man im Rahmen dieses Tests hingegen zu dem Ergebnis, dass die Haftung in gleicher Gestalt und damit ungeachtet der konkreten Parteiabrede besteht, ist diese Haftung deliktisch zu qualifizieren und damit dem Kollisionsrechtsregime der Rom II-Verordnung zuzuweisen. Denn bei funktionaler Betrachtung besteht dann kein Grund, der die unterschiedliche kollisionsrechtliche Handhabung derartiger Schadensersatzansprüche rechtfertigen würde. ${ }^{34}$ Es handelt sich dann ja gerade nicht um eine Situation, in welcher der Schädiger gegenüber dem Geschädigten eine Pflicht verletzt, die lediglich deshalb bestand, weil Schädiger und Geschädigter kraft ihrer vereinten Willkür ein vertragliches Band geschaffen hatten. ${ }^{35}$ Vielmehr verletzt der Schädiger eine Pflicht, die ihm schon von Gesetzes wegen und nicht erst aufgrund einer parteiautonom getroffenen Vereinbarung obliegt, wenngleich sich diese im vertraglichen Gewand präsentiert. Es geht in derartigen Fällen also nicht um die Anerkennung der Privatautonomie auf der Ebene des Kollisionsrechts, sondern um die Durchsetzung gesetzlicher Vorgaben zum Schutz des status quo der Vertragsparteien.

Handlungen, die eine unabhängig von einer privatautonomen Vereinbarung bestehende, gesetzliche Pflicht verletzen, sind nach alledem ausschließlich als Delikt zu qualifizieren und somit dem Kollisionsrechtsregime der Rom II-Verordnung zuzuordnen. ${ }^{36}$ Für eine (zusätzliche) vertragliche Qualifikation und eine damit einhergehende Betonung der Privatautonomie auf der Ebene des Kollisionsrechts besteht weder ein Grund noch eine Rechtfertigung: ${ }^{37}$ Die Idee eines Vertrags auf der Ebene des Sachrechts ist ja gerade, dass sich die Vertragsschließenden kraft ihres Willens zu einer Leistung verpflichten, die sie kraft Gesetzes nicht zu erbringen hätten. Der Gläubiger erhält aufgrund des Vertrags ein Forderungsrecht gegenüber dem Schuldner, wodurch das Äquivalenzinteresse des Gläubigers entsteht. Das Äquivalenzinteresse, welches hier auf einer ersten Stufe zur Abgrenzung von Vertrag und Delikt herangezogen wird, ist also letztlich Auswuchs der Privatautonomie. Bezüglich der vereinbarten Leistung und deren Erbringung gilt das vereinbarte Rege-

30 Vgl. den ursprünglichen Vorschlag der Kommission für eine Verordnung des Europäischen Parlaments und des Rates über das auf außervertragliche Schuldverhältnisse anzuwendende Recht („Rom II“), KOM 2003 (427) endg., S. 13.

31 Ursprünglicher Vorschlag der Kommission für eine Verordnung des Europäischen Parlaments und des Rates über das auf außervertragliche Schuldverhältnisse anzuwendende Recht („Rom II“), KOM 2003 (427) endg., S. 13.

32 Nordmeier, in: Gebauer/Wiedmann (Hrsg.), Zivilrecht unter europäischem Einfluss, 2. Auflage 2010, Kap. 37 Rn. 8; Lehmann, (Fn. 12), 17, 29 f.

33 Vgl. Jarvis v. Moy, Davis, Smith, Vandervell \& Co., (1936) 1 K. B. 399, 405; Prosser/Keeton, (Fn. 19), $\$ 92$ S. 656; Yeager v. Dunnavan, 176 P.2d 755, 757, Supreme Court, Washington (1946); G. W. Construction Corporation v. Professional Service Industries, 70 Wash.App. 360, 364, 853 P.2d 484, 486, Court of Appeals, Washington (1993); Leicht, (Fn. 15), S. 154; vgl. auch Unberath, (Fn. 15), S. 191, insb. Fn. 44; Schlosser, IPRax 1984, 65, 67; Magnus, in: Staudinger, Neubearbeitung 2011, Art. 1 Rom I Rn. 33.

34 Vgl. Kurt, Culpa in contrahendo im europäischen Kollisionsrecht der vertraglichen und außervertraglichen Schuldverhältnisse, 2009, S. 78; Leicht, (Fn. 15), S. 155.

35 Vgl. dazu Dickinson, (Fn. 4), Rn. 3.113 und Rn. 3.129; Prosser/Keeton, (Fn. 19), § 92 S. 655; Kaye, Civil Jurisdiction, S. 562 und S. 563; Spelsberg-Korspeter, Anspruchskonkurrenz im internationalen Privatrecht, 2009, S. 65, 134; Leicht, (Fn. 15), S. 155; Schurig, in: FS Heldrich, 1021, 1027; Lohse, Das Verhältnis von Vertrag und Delikt, 1991, S. 214; Schlosser, IPRax 1984, 65, 67; Bröcker, (Fn. 23), S. 197; Cheshire/North/Fawcett, Private International Law, 14. Auflage 2008, S. 678.

36 Base Metal Trading Ltd. v. Shamurin [2004] EWCA Civ 1316 at para 28 (L.J. Tuckey); Dickinson, (Fn. 35), Rn. 3.129, 3.131; vgl. auch Leicht, (Fn. 15), S. 155 und Kropholler/Von Hein, (Fn. 8), Art. 5 EuGVO Rn. 16 i.V.m. Rn. 74 am Anfang.

37 A.A. Magnus, in: Staudinger, (Fn. 33), Art. 1 Rom I Rn. 32 welcher - leider ohne nähere Begründung - der Meinung ist, dass ein Ausschlussverhältnis zwischen Vertrag und Delikt im Kollisionsrecht nicht angebracht sei und daher an sich deliktische Ansprüche als auch vertraglich qualifizieren möchte. Insoweit setzt er sich zu der hiesigen Auffassung aber auch zu seiner eigenen Aussage in Staudinger, (Fn. 33), Einl. zur Rom I Rn. 30 wonach die Rom I und II-Verordnung jeweils ihren eigenen Anwendungsbereich hätten und alle Schuldverhältnisse, die nicht als vertraglich einzustufen sind unter die Rom II-Verordnung fallen, in Widerspruch. 
lungswerk, weil die Vertragsschließenden dessen Geltung vereinbart haben. ${ }^{38}$ Erkennt man die zentrale Bedeutung der Privatautonomie beziehungsweise des freien Willens der Parteien für den Vertrag und die daraus resultierenden Pflichten auf der Ebene des Sachrechts, ist es nur folgerichtig ihr auch auf der Ebene des Kollisionsrechts diese Schlüsselrolle zukommen zu lassen. ${ }^{39}$ Existieren bestimmte Pflichten der Parteien hingegen nicht erst aufgrund eines Vertrages, sondern bereits aufgrund einer gesetzlichen Anordnung und damit unabhängig von einer irgendwie gearteten freiwilligen Vereinbarung durch die Parteien, ${ }^{40}$ fällt die innere Rechtfertigung für eine Rechtswahlmöglichkeit, nämlich die Anerkennung der Privatautonomie auf kollisionsrechtlicher Ebene, zumindest partiell weg. In der Folge wäre es in diesen Fällen verfehlt, wenn man die Parteiautonomie durch eine vertragliche Qualifikation betonen würde.

Dieser Art der Abgrenzung anhand der Funktion von Vertrag und Delikt kann auch nicht entgegengehalten werden, dass sowohl die objektivierten Vertragsinhalte, also das standardisierte Vertragsprogramm der Parteien, als auch die deliktischen Verhaltenspflichten der Rechtsordnung entstammen und daher nicht trennbar sind. ${ }^{41}$ Auch die Erwägung, dass die Privatautonomie auf der kollisionsrechtlichen Ebene durch die Vornahme einer vertraglichen Qualifikation bereits dann zu schützen sei, wenn die durch den Schädiger konkret verletzte gesetzliche Pflicht nur deshalb besteht, weil er die Garantenstellung freiwillig übernommen hat, ${ }^{42}$ vermag nicht zu überzeugen. Pflichten, die aus einer einseitigen freiwilligen Übernahme einer Garantenstellung resultieren, sind nicht Auswuchs der Privatautonomie, sondern bestehen kraft gesetzlicher Anordnung, so dass es verfehlt wäre, die Privatautonomie auf der Ebene des Kollisionsrechts übermäßig zu betonen. Dass eine ausschließlich deliktische Qualifikation von Integritätsinteressen auch innerhalb eines vertraglichen Bandes sowohl mit der Rechtsprechung des EuGH und des BGH, als auch mit der Existenz der Ausweichklausel des Art. 4 Abs. 3 S. 2 Rom II vereinbar ist, wurde bereits an anderer Stelle gezeigt ${ }^{43}$.

\section{Zwischenergebnis}

Zusammenfassend bedeutet dies, dass im europäischen Kollisionsrecht in einem ersten Schritt die Unterscheidung zwischen dem Leistungsinteresse auf der einen und dem Bestandsschutzinteresse auf der anderen Seite für die Qualifikation eines Anspruchs als vertraglich oder deliktisch entscheidend ist. Gelangt man bei dieser Unterscheidung zu einem eindeutigen Ergebnis, kann man es bei diesem Schritt belassen. Anderenfalls ist in einem zweiten Schritt zu prüfen, ob es schwerpunktmäßig um die Anerkennung der Parteiautonomie und die Anwendung des Rechts der die vertragscharakteristischen Leistung erbringenden Partei geht. Ist dies der Fall, ist ausschließlich eine vertragliche Qualifikation vorzunehmen. Geht es hingegen um einen angemessenen Ausgleich der kollisionsrechtlichen Interessen von Täter und Opfer sowie die Voraussehbarkeit der anwendbaren Sicherheits- und Verhaltensregeln, erscheint eine deliktische Qualifikation sach- und interessengerecht.

\section{Anwendung auf die in der GEK geregelte Haftung wegen Sach- oder Personenschäden}

Für die hier ins Zentrum der Untersuchung gerückten Sachoder Personenschäden bedeutet dies folgendes:

\section{Haftung für Personenschäden infolge der Mangelhaftigkeit der Kaufsache}

Die Haftung des Verkäufers für Personenschäden, die aus einer mangelhaften Kaufsache resultieren, ist im GEK in den Art. 159, 87 geregelt. $^{44}$

Für die Qualifikation dieser Ansprüche ist entsprechend des hier vorgeschlagenen 2-stufigen Tests auf erster Stufe zu untersuchen, ob der Verkäufer durch die Lieferung der mangelhaften Sache, die dann zu einem Körper- oder Gesundheitsschaden führt, das Äquivalenz- oder das Integritätsinteresse des Käufers verletzt.

Haftungsauslösend ist in diesen Fällen nicht der Umstand, dass der Verkäufer eine Leistungspflicht und damit das Äquivalenzinteresses des Käufers verletzt, sondern die Verletzung einer gesetzlichen Pflicht zum Schutz der körperlichen Integrität des Käufers und damit die Verletzung des Integritätsinteresses. Es geht bei dieser Haftung nicht um den Schutz des Interesses des Käufers am Erhalt einer gebrauchsfähigen Ware. Vielmehr zielt diese Haftung im Wesentlichen darauf ab, dass der bereits im Zeitpunkt des Vertragsschlusses bestehende status quo an Rechtsgütern des Käufers gesichert wird. Da folglich die Verletzung des Integritätsinteresses des Käufers durch den Verkäufer haftungsauslösend ist, ist die Haftung des Verkäufers ausschließlich als deliktisch zu qualifizieren.

Dass es sich bei dieser Haftung des Verkäufers ausschließlich um eine solche wegen Verletzung des Integritätsinteresses des Käufers handelt, wird freilich von der Warte des deutschen Sachrechts allzu leicht übersehen, da zahlreiche Stimmen die Pflichtverletzung in diesen Fällen ausschließlich in der Lieferung einer mangelhaften Kaufsache sehen. ${ }^{45}$ Danach sei die Verletzung der Primärpflicht des Käufers, eine mangelfreie Sache zu liefern, haftungsauslösend. Bei genauerem Hinsehen zeigt sich jedoch, dass der Integritätsschaden gerade nicht dadurch entstanden ist, dass der Käufer nicht entsprechend seines primären Pflichtenprogramms aus Art. 91 lit. a), lit. c) GEK mangelfrei geleistet hat,

38 Flume, Allgemeiner Teil des Bürgerlichen Rechts, Band 2: Das Rechtsgeschäft, 4. Auflage 1992, S. 7.

39 Martiny, in: MüKo, (Fn. 4), Art. 3 R I Rn. 8; Leicht, (Fn. 15), S. 155.

40 Der Geltungsgrund der vertraglichen Haftung besteht dann nicht in einem Leistungsversprechen als Akt autonomer Selbstbindung der Parteien, sondern vielmehr - im Prinzip wie bei der deliktischen Haftung - auf einem willensunabhängigen Befehl des Gesetzes. Insoweit ist in diesen Fällen Picker, JZ 1987, 1041, 1044 zuzustimmen; vgl. auch Leicht, (Fn. 15), S. 155.

41 Vgl. hierzu ausführlich Wendelstein, (Fn. 2), S. 160.

42 So Rusworth/Scott, LMCLQ 2008, 274, 300.

43 Wendelstein, (Fn. 2), S. 183-194.

44 Im deutschen Sachrecht finden sich diese Ansprüche richtigerweise grundsätzlich in den $\$ \$ 280$ Abs. 1, 241 Abs. 2 und $\$ 823$ Abs. 1 BGB. Vgl. dazu etwa Canaris, ZIP 2003, 321, 324; Ehmann/Sutschet, JZ 2004, 62, 70; Katzenstein, JURA 2004, 800, 804 f.; Richardi, NZA 2002, 2004, 2011.

45 Statt vieler nur Weidekaff, in: Palandt, (Fn. 4), $\$ 437$ BGB Rn. 35; Lorenz, NJW 2002, 2497, 2500. 
sondern vielmehr dadurch, dass er eine schadensträchtige Sache geliefert hat. Hierdurch hat er eine integritätsschützende vertragliche Neben- bzw. Schutzpflicht im Sinne des Art. 87 Abs. 1 GEK $^{46}$ verletzt, die ihn gemäß Art. 87 Abs. 1, Art. 3, 159 GEK $^{47}$ verschuldensunabhängig zum Schadensersatz verpflichtet, sofern nicht Art. 88 GEK oder Art. 161 GEK $^{48}$ eingreift. ${ }^{49}$

Festzuhalten gilt es, dass die Haftung für Personenschäden infolge der Mangelhaftigkeit der Kaufsache lediglich dem Schutz des Integritätsinteresses des Käufers dient und damit ausschließlich als deliktisch zu qualifizieren ist. Derartige Ansprüche unterfallen daher dem sachlichen Anwendungsbereich der Rom IIVerordnung.

Da das Ergebnis eindeutig ist, ist es an sich nicht nötig mit der zweiten Stufe des hier vorgeschlagenen Test fortzufahren, weshalb nur kurz auf sie eingegangen wird: Auf der zweiten Stufe ist zu überprüfen, ob bei gleicher Faktizität der Ereignisse ohne freiwillig eingegangene Verpflichtung, also ohne privatautonome Abrede, keine Haftung bestehen würde. Hat der Verkäufer die Lieferung der schadensträchtigen Kaufsache verschuldet, kann man den Kaufvertrag wegdenken, ohne dass die Haftung des Verkäufers für aus dieser Pflichtverletzung resultierende Personenschäden entfiele. Die Haftung bestünde, sofern Deliktsstatut deutsches Recht ist, nach wie vor gemäß $\$ 823$ Abs. 1 BGB, da die persönliche Integrität als absolutes Rechtsgut dem deliktischen Schutz untersteht. Die Haftung des Verkäufers resultiert also nicht aus einer aufgrund der Privatautonomie getroffenen Vereinbarung von Käufer und Verkäufer, sondern aus der Verletzung der gesetzlichen Pflicht, dass keine schadensträchtigen Sachen in den Verkehr gebracht werden dürfen. Für eine Anerkennung der Privatautonomie auf kollisionsrechtlicher Ebene durch eine vertragliche Qualifikation besteht daher kein Grund. Schließlich führt auch der entwickelte funktionale Vertragsbegriff dazu, dass die Haftung für Personenschäden infolge der Schadensträchtigkeit der Kaufsache ausschließlich als deliktisch $\mathrm{zu}$ qualifizieren ist.

\section{Haftung für Sachschäden infolge der Mangelhaftigkeit an Gegenständen außerhalb der Kaufsache}

Auch die Haftung für Sachschäden an kaufgegenstandsfremden Sachen, die aus der Mangelhaftigkeit der Kaufsache resultieren, ist in 87 Abs. 1, Art. 3, 159 GEK geregelt. ${ }^{50}$ Für die Qualifikation dieser Ansprüche als ausschließlich deliktisch kann entsprechend den obigen Ausführungen zu den Personenschäden folgendes festgestellt werden: Haftungsauslösend ist in diesen Fällen nicht der Umstand, dass der Verkäufer seine Primärpflicht aus Art. 91 GEK und damit das Äquivalenzinteresses des Käufers verletzt, sondern einzig die Verletzung einer gesetzlichen Pflicht (Art. 87 Abs. 1, 3 GEK) zum Schutz des Integritätsinteresses des Käufers. Diese Haftung zielt darauf ab, dass der bereits im Zeitpunkt des Vertragsschlusses bestehende status quo des Käufers gesichert wird. Bezeichnenderweise haftet der Verkäufer in diesen Fällen, sofern er die Schadensträchtigkeit der Kaufsache zu vertreten hat, bei deutschem Deliktsstatut gemäß $\$ 823$ Abs. 1 BGB. Die parteiautonome Vereinbarung ist also nicht conditio sine qua non für die Haftung des Verkäufers, so dass für eine Betonung der Privatautonomie auf der Ebene des Kollisionsrechts weder ein Grund noch eine Rechtfertigung besteht. Viel- mehr sind derartige Ansprüche des Käufers gegen den Verkäufer ausschließlich als deliktisch zu qualifizieren und somit dem Kollisionsrechtsregime der Rom II-Verordnung zuzuweisen.

\section{Haftung für Sachschäden an der Kaufsache selbst infolge deren Mangelhaftigkeit}

Wird die Kaufsache infolge deren Mangelhaftigkeit beschädigt oder zerstört, sind daraus resultierende Schadensersatzansprüche des Käufers gegen den Verkäufer ausschließlich als vertraglich zu qualifizieren. ${ }^{51}$ Der Käufer wird in derartigen Fällen einzig in seinem Äquivalenzinteresse verletzt, da ihm zum Zeitpunkt des Vertragsschlusses die Kaufsache (noch) nicht als Rechtsgut durch die Rechtsordnung zugewiesen war. Durch die Lieferung einer mangelhaften Kaufsache wird der Käufer demnach nicht in dem status quo seiner Rechte und Rechtsgüter verletzt. Vielmehr geht es bei der Haftung für Sachschäden infolge der Mangelhaftigkeit an der Kaufsache selbst ausschließlich um den Schutz des Interesses des Käufers am Erhalt einer gebrauchsfähigen Ware und damit um den Schutz des Äquivalenzinteresses des Käufers.

\section{Konsequenzen für die Vorschaltlösung}

Die Untersuchung hat ergeben, dass die Haftung des Verkäufers für Sachschäden, die infolge der Mangelhaftigkeit der Kaufsache außerhalb der Kaufsache auftreten, ausschließlich als außervertraglich zu qualifizieren ist und daher dem Kollisionsrechtsregime der Rom II-Verordnung unterstehen. Gleiches gilt für Ansprüche des Käufers gegen den Verkäufer für Schädigungen seiner körperlichen Integrität infolge der Mangelhaftigkeit der Kaufsache. Dies bedeutet, dass man in diesen Fällen nicht zur Anwendung des GEK gelangen kann, da eine „Binnenwahl“ zugunsten des GEK innerhalb der über das Kollisionsrechtsregime der Rom II-Verordnung zur Anwendung berufenen Sachrechtsordnung eines Mitgliedstaates gemäß Erwägungsgrund 10 zum GEK ausscheidet. ${ }^{52}$

Für die im GEK geregelten Ansprüche wegen Personenschäden infolge der Mangelhaftigkeit der Kaufsache würde dies bedeuten, dass diese in keinem Fall zur Anwendung berufen sein

\footnotetext{
46 Vgl. dazu Lorenz, AcP 212 (2012), 702, 744.

47 Mehrheitlich wird davon ausgegangen, dass durch das GEK nicht nur das Erfüllungsinteresse sondern auch das Integritätsinteresse geschützt wird, so dass auch Mangelfolgeschäden an sonstigen Rechtsgütern des Käufers nach den Art. 159 ff. GEK ersetzbar sind. Vgl. dazu Lorenz, AcP 212 (2012), 702, 795; Kieninger, (Fn. 1), 205, 211.

48 Vgl. dazu Kieninger, (Fn. 1), 205, $211 \mathrm{f}$.

49 Für Verletzungen von Leistungspflichten durch Schlechtleistung, die darüber hinaus zur Verletzung des Integritätsinteresses des Käufers führen (etwa durch Verletzung seiner körperlichen Integrität als „Mangelfolgeschaden“) bedeutet dies im deutschen Recht: Ersatz des Gesamtschadens am Leistungs- und Integritätsinteresse kann der Käufer nur auf der Grundlage zweier verschiedener Haftungstatbestände verlangen. Vgl. dazu Keuk, Vermögensschaden und Interesse, 1972, S. 163 f.; Katzenstein, JURA 2004, 800, 804; ders., Haftungsbeschränkungen zugunsten und zulasten Dritter, S. $177 \mathrm{f}$.

50 Im deutschen BGB sind richtigerweise die $\$ \$ 280$ Abs. 1, 241 Abs. 2 BGB sowie $\$ 823$ Abs. 1 BGB einschlägig.

51 Vgl. von Hein, RIW 2000, 820, 821.

52 Vgl. dazu die in der Einleitung gemachten Ausführungen.
} 
können. Eine Lösung dieses kaum gewollten Ergebnisses könnte darin bestehen, dass man den Wortlaut des Erwägungsgrunds 10 zum GEK nicht allzu ernst nimmt, sondern vielmehr die Rom Iund II-Verordnungen entsprechend ihres sachlichen Anwendungsbereichs anwendet und im Fall, dass über deren Kollisionsregeln das Recht eines Mitgliedstaates zur Anwendung berufen ist, überprüft, ob ein wirksames opt-in in das GEK erfolgt ist oder nicht. Hierzu müsste man sich jedoch über den an sich klaren Wortlaut des Erwägungsgrundes 10 zum GEK hinwegsetzen. Obwohl dies grundsätzlich möglich ist, weil die Erwägungsgründe nicht verbindlich sind, wäre eine Entscheidung des europäischen Gesetzgebers vorzugswürdig. Solange eine Änderung des Wortlauts noch möglich ist, sollte die Kommission ihre Chance zur Änderung des Erwägungsgrundes 10 zum GEK nutzen! Eine solche Änderung könnte etwa dergestalt erfolgen, dass der Passus „in Bezug auf vorvertragliche Informationspflichten“ in Erwägungsgrund 10 zum GEK ersatzlos gestrichen wird.

\section{Fazit}

Die europäische Kommission hat es in ihrem Entwurf eines Gemeinsamen europäischen Kaufrechts bisher versäumt, die Grenze zwischen Vertrag und Delikt in gleicher Art und Weise zu ziehen, wie sie im Rahmen des europäischen Internationalen Privatrechts gezogen wird. Deswegen kann die von der Kommission befürwortete Vorschaltlösung nicht in allen Fällen wie angedacht funktionieren.

Das eigentliche Problem liegt jedoch tiefer: Solange der europäische Gesetzgeber seine Systembegriffe in den unterschiedlichen Regelungsakten nicht einheitlich ausgestaltet, wird es ihm nicht gelingen ein homogenes Regelungsregime zu schaffen. Bezeichnend sind insoweit die hier untersuchten Systembegriffe „Vertrag“ und „Delikt“: Der Grund für die Ausbildung eines Vertrags- und Deliktsstatuts liegt darin, dass aus der unterschiedlichen Funktion von Vertrag und Delikt auf der Ebene des Sachrechts unterschiedliche kollisionsrechtliche Interessenlagen resultieren. Regelt der europäische Gesetzgeber mit dem GEK nun das Sachrecht und definiert auf dieser Ebene unterschiedliche Ansprüche als vertraglich, bestimmt er damit indirekt auch, welche Ansprüche auf der Ebene des europäischen Kollisionsrechts als vertraglich zu qualifizieren sind. Da im europäischen Vertragsbegriff letztlich der Schlüssel zur Abgrenzung von vertraglichen und deliktischen Ansprüchen liegt, sollte sich der europäische Gesetzgeber gut überlegen, ob sich seine Regelungen auf der Ebene des Sachrechts in das bereits bestehende System des europäischen Zivilprozessrechts und des europäischen Kollisionsrechts einfügen. Nur wenn dies der Fall ist, kann ein tragfähiges, anwenderfreundliches und Rechtssicherheit schaffendes Gesamtsystem entstehen. Letztlich sollte sich der europäische Gesetzgeber überlegen, ob es nicht an der Zeit wäre, die Schlüsselsystembegriffe wie beispielsweise den Vertrag einheitlich für alle Verordnungen und Richtlinien zu definieren. So manche Zweifelsfrage würde hierdurch obsolet. Insoweit sei abschließend darauf hingewiesen, dass eine Definition der Schlüsselbegriffe im DCFR mangels gesetzgeberischer Legitimation dieses Werkes nicht genügt. Aus dem selben Grund dürfen die dortigen Definitionen auch nicht als Auslegungshilfe verwendet werden. ${ }^{53}$

\section{Zusammenfassung}

Nach dem Willen der Kommission soll die Vereinbarung über die Verwendung des GEK eine Wahl sein, die innerhalb des einzelstaatlichen Rechts getroffen wird, das nach der Rom I-Verordnung beziehungsweise in Bezug auf vorvertragliche Informationspflichten nach der Verordnung Rom II-Verordnung anwendbar ist. Es soll sich bei der Wahl des GEK daher nicht um eine Wahl im Sinne des Kollisionsrechts handeln. Wie in dem Beitrag gezeigt wurde enthält das GEK Schadensersatzansprüche für Mangelfolgeschäden. Diese sind zwar als deliktisch im Sinne der Rom II-Verordnung zu qualifizieren, weisen aber keinerlei Bezug zu vorvertraglichen Informationspflichten auf. Folglich versagt insoweit die von der Kommission gewollte kollisionsrechtliche Vorschaltlösung.

\section{Summary}

According to the Commission's concept the agreement on the use of the CESL should be a choice exercised within the scope of the respective national law which is applicable pursuant to the Rome I Regulation or, in relation to pre-contractual information duties, pursuant to the Rome II Regulation. The agreement on the use of the CESL should therefore not amount to, and not be confused with, a choice of the applicable law within the meaning of the conflict-of-law rules. As demonstrated in this article the scope of application of the CESL comprises claims for consequential harm caused by a defect. Such claims can indeed be characterised as delictual in the sence of the Rome II Regulation even though they do not have any relation to pre-contractual duties to inform referred to in the CESL. Consequently the concept of CESL as a 2 nd national regime fails at least in this regard.

\section{Résumé $^{*}$}

Par référence au concept de la Commission, l'accord concernant l'utilisation du droit commun européen de la vente doit être un choix exercé dans les limites du droit national, qui est applicable selon le règlement Rome I, respectivement, en cas d'obligations précontractuelles d'information, selon le règlement Rome II. Le choix du droit commun européen de la vente n'est donc pas, selon le concept de la Commission, un choix dans le sens du droit international privé. Comme indiqué dans cet article, le droit commun européen de la vente contient des provisions concernant des dommages causés par une pièce défectueuse. Il est vrai que ces droits doivent être qualifiés comme délictuel selon le règlement Rome II, mais ils n'ont pas de rapport aux obligations précontractuelles d'information. Par conséquent, la solution de la Commission de créer un deuxième régime national ne fonctionne pas par rapport à cette question.

\footnotetext{
53 Stürner, in: Schulte-Nölke/Zoll/Jansen/Schulze, Der Entwurf für ein optionales europäisches Kaufrecht, 2012, 47, 77 f.

* Übersetzt von Christoph Fischer.
} 